\title{
Fractional Hamiltonian of Nonconservative Systems with Second Order Lagrangian
}

\author{
Ola Jarab'ah ${ }^{1}$, Khaled Nawafleh ${ }^{2}$ \\ ${ }^{1}$ Applied Physics Department, Faculty of Science, Tafila Technical University, Tafila, Jordan \\ ${ }^{2}$ Department of Physics, Faculty of Science, Mu'tah University, AL-Karak, Jordan
}

Email address:

oasj85@yahoo.com (O. Jarab'ah)

\section{To cite this article:}

Ola Jarab'ah, Khaled Nawafleh. Fractional Hamiltonian of Nonconservative Systems with Second Order Lagrangian. American Journal of Physics and Applications. Vol. 6, No. 4, 2018, pp. 85-88. doi: 10.11648/j.ajpa.20180604.12

Received: July 5, 2018; Accepted: July 19, 2018; Published: August 24, 2018

\begin{abstract}
In this paper, the nonconservative systems with second order Lagrangian are investigated using fractional derivatives. The fractional Euler Lagrange equations for these systems are obtained. Then, fractional Hamiltonian for these systems is constructed, which is used to find the Hamilton's equations of motion in the same manner as those obtained by using the formulation of Euler Lagrange equations from variational problems, and it is observed that the Hamiltonian formulation is in exact agreement with the Lagrangian formulation. The passage from the Lagrangian containing fractional derivatives to the Hamiltonian is achieved. We have examined one example to illustrate the formalism.
\end{abstract}

Keywords: Fractional Derivatives, Lagrangian Formulation, Hamiltonian Formulation, Nonconservative Systems, Euler Lagrange Equations, Second Order Lagrangian

\section{Introduction}

The fractional derivatives have played a significant role in physics, mathematics and engineering [1-4]. Several attempts have been made to include nonconservative forces in the Lagrangian and Hamiltonian mechanics. Riewe $[5,6]$ presented a new approach to mechanics that allows one to obtain the equations for nonconservative systems using fractional derivatives. Eqab et al., [7, 8] developed a general formula for determining the potentials of arbitrary forces, conservative and nonconservative using the Laplace transform of fractional integrals and fractional derivatives and also they constructed the Hamiltonian formulation of discrete and continuous fields in term of fractional derivatives [9].

The formalism for investigating the fractional variational problem of Lagrange represents an important part of fractional calculus and it was discussed by Agrawal $[10,11]$ and this formalism can be extended to Lagrangian systems with higher derivatives. Recently Euler Lagrange equations have been presented for unconstrained and constrained fractional variational problems by Agrawal [12]. The resulting equations are found to be similar to those for variational problems containing integral order derivatives. In other words, the results of fractional calculus of variations reduce to those obtained from traditional fractional calculus of variations when the derivative of fractional order replaced by integral order. This approach is extended to classical fields with fractional derivatives [13]. In addition the problem of having nonconservative equations of motion arise from the use of a variational principle is reexamined by Dreisi-gmeyer and Yoang [14]. The action is treated as a Volterra series. Besides, the modeled described by fractional order derivatives of Riemann-Liouville type in sequential form are discussed in Lagrangian and Hamiltonian formalism by Klimek [15]. In her work, Klimek showed that the fractional Hamiltonian is usually not a constant of motion, even in the case when the Hamiltonian is not an explicit function of time. While in the limit the fractional operators give classical derivatives of the first order, the total time derivatives of the Hamiltonian coincide with the partial one.

More recently, the fractional Hamiltonian analysis for higher order derivatives systems were investigated for nonsingular systems and the generalization of Ostrogradski's formulation was discussed with in framework of fractional calculus [16]. The Hamilton-Jacobi formulation for discrete Lagrangian systems containing second order fractional 
derivatives was discussed by Hasan [17].

In the present paper as a continuation of previous works, the generalized mechanics is considered to obtain the Euler Lagrange equations and Hamilton's equations of motion for nonconservative systems with second order Lagrangians depending on fractional derivatives of coordinates.

The paper is organized as follows: In Section 2, Agrawal's work fractional Lagrangian mechanics is reviewed briefly. In Section 3, the fractional Hamiltonian of nonconservative systems with second order Lagrangian is constructed. In Section 4, one illustrative example is examined. The work closes with some concluding remarks (Section 5).

\section{Agrawal's Fractional Lagrangian Mechanics}

In Agrawal's work [12] the problem is formulated in term of the left and the right Riemann Liouville fractional derivatives, which is defined as [18].

The left Riemann Liouville fractional derivative reads as

$$
{ }_{a} D_{x}^{\alpha} f(x)=\frac{1}{\Gamma(n-\alpha)}\left(\frac{d}{d x}\right)^{n} \int_{a}^{x}(x-\tau)^{n-\alpha-1} f(\tau) d \tau
$$

which is denoted as the LRLFD, and the right Riemann Liouville fractional derivative reads as

$$
{ }_{x} D_{b}^{\alpha} f(x)=\frac{1}{\Gamma(n-\alpha)}\left(-\frac{d}{d x}\right)^{n} \int_{x}^{b}(\tau-x)^{n-\alpha-1} f(\tau) d \tau
$$

which is denoted as the RRLFD.

Here $\Gamma$ represents the Euler's gamma function, and $\alpha$ is the order of the derivative such that $n-1 \leq \alpha<n$ and is not equal to zero. If $\alpha$ is an integer, these derivatives are defined as follows

$$
\begin{gathered}
{ }_{a} D_{x}^{\alpha} f(x)=\left(\frac{d}{d x}\right)^{\alpha} f(x) \\
{ }_{x} D_{b}^{\alpha} f(x)=\left(-\frac{d}{d x}\right)^{\alpha} f(x) \\
\alpha=1,2, \ldots
\end{gathered}
$$

The RL fractional derivatives have the general properties can be written as

$$
{ }_{a} D_{t}^{p}\left({ }_{a} D_{t}^{-q} f(t)\right)={ }_{a} D_{t}^{p-q} f(t)
$$

under the assumptions that $f(t)$ is continuous and $p \geq q \geq 0$.

For $p>0$ and $t>\mathrm{a}$, we get

$$
{ }_{a} D_{t}^{p}\left({ }_{a} D_{t}^{-p} f(t)\right)=f(t)
$$

$$
{ }_{a} D_{t}^{\alpha}\left({ }_{a} D_{t}^{\beta} f(t)\right)={ }_{a} D_{t}^{\alpha+\beta} f(t)
$$

Let $f$ and $g$ are two continuous functions on $[a, b]$. Then, for all $t \in[a, b]$, the following property holds:

for

$$
m>0 \quad, \int_{a}^{b}\left({ }_{a} D_{t}^{m} f(t)\right) g(t) d t=\int_{a}^{b} f(t)\left({ }_{t} D_{b}^{m} g(t)\right) d t
$$

The Euler Lagrange equations for the fractional calculus of variations problem with second order derivatives are obtained as

$$
\begin{aligned}
& \frac{\partial L}{\partial q}+{ }_{t} D_{b}^{\alpha} \frac{\partial L}{\partial_{a} D_{t}^{\alpha} q}+{ }_{a} D_{t}^{\beta} \frac{\partial L}{\partial_{t} D_{b}^{\beta} q}+{ }_{t} D_{b}^{2 \alpha} \frac{\partial L}{\partial_{a} D_{t}^{2 \alpha} q} \\
& +{ }_{a} D_{t}^{2 \beta} \frac{\partial L}{\partial_{t} D_{b}^{2 \beta} q}=0
\end{aligned}
$$

Here, $\mathrm{L}$ is a function of the form:

$$
L=L_{\circ}\left(q,{ }_{a} D_{t}^{\alpha} q,{ }_{t} D_{b}^{\beta} q,{ }_{a} D_{t}^{2 \alpha} q,{ }_{t} D_{b}^{2 \beta} q\right) e^{\gamma t},
$$

which describes the irregular Lagrangian or (nonconservative system) and $\gamma$ is defined as damping factor [19].

For $\alpha=\beta=1$, we have

$$
{ }_{a} D_{t}^{\alpha}=\frac{d}{d t}
$$

and

$$
{ }_{t} D_{b}^{\alpha}=-\frac{d}{d t}
$$

and equation (9) reduces to the standard Euler Lagrange equation of second order Lagrangian.

\section{Fractional Hamiltonian of Non-conservative Systems}

For nonconservative systems consider the Lagrangian depending on the fractional time derivatives of coordinates in the form

$$
L=L_{\circ}\left(q,{ }_{a} D_{t}^{\alpha} q,{ }_{t} D_{b}^{\beta} q,{ }_{a} D_{t}^{2 \alpha} q,{ }_{t} D_{b}^{2 \beta} q\right) e^{\gamma t}
$$

We introduce the fractional generalized momenta as [20]

$$
\begin{gathered}
p_{\alpha}=\frac{\partial L}{\partial_{a} D_{t}^{\alpha} q}-{ }_{a} D_{t}^{\alpha}\left(\frac{\partial L}{\partial_{a} D_{t}^{2 \alpha} q}\right) \\
p_{\beta}=\frac{\partial L}{\partial_{t} D_{b}^{\beta} q}+{ }_{t} D_{b}^{\beta}\left(\frac{\partial L}{\partial_{t} D_{b}^{2 \beta} q}\right)
\end{gathered}
$$

The general formula of semi group property is written as [2] 


$$
\begin{gathered}
\pi_{\alpha}=\frac{\partial L}{\partial_{a} D_{t}^{2 \alpha} q} \\
\pi_{\beta}=\frac{\partial L}{\partial_{t} D_{b}^{2 \beta} q}
\end{gathered}
$$

and the Hamiltonian depending on the fractional time derivatives reads as

$$
\begin{aligned}
& H=p_{\alpha a} D_{t}^{\alpha} q+p_{\beta t} D_{b}^{\beta} q+\pi_{\alpha a} D_{t}^{2 \alpha} q \\
& +\pi_{\beta{ }_{t}} D_{b}^{2 \beta} q-L
\end{aligned}
$$

Calculating the total differential of this Hamiltonian we obtain

$$
\begin{aligned}
& d H=p_{\alpha} d_{a} D_{t}^{\alpha} q+d p_{\alpha a} D_{t}^{\alpha} q+p_{\beta} d_{t} D_{b}^{\beta} q+d p_{\beta} D_{b} D_{b} q \\
& +\pi_{\alpha} d_{a} D_{t}^{2 \alpha} q+{ }_{a} D_{t}^{2 \alpha} q d \pi_{\alpha}+\pi_{\beta} d_{t} D_{b}^{2 \beta} q+{ }_{t} D_{b}^{2 \beta} q d \pi_{\beta} \\
& -\frac{\partial L}{\partial q} d q-\frac{\partial L}{\partial_{a} D_{t}^{\alpha} q} d_{a} D_{t}^{\alpha} q-\frac{\partial L}{\partial_{t} D_{b}^{\beta} q} d_{t} D_{b}^{\beta} q \\
& -\frac{\partial L}{\partial_{a} D_{t}^{2 \alpha} q} d_{a} D_{t}^{2 \alpha} q-\frac{\partial L}{\partial_{t} D_{b}^{2 \beta} q} d_{t} D_{b}^{2 \beta} q-\frac{\partial L}{\partial t} d t
\end{aligned}
$$

Substituting the values of the momenta from equations (11-14) in equation (16) and making use of the Euler Lagrange equation (9), we obtain

$$
\begin{aligned}
& d H=d p_{\alpha a} D_{t}^{\alpha} q+d p_{\beta} D_{b}^{\beta} q+{ }_{a} D_{t}^{2 \alpha} q d \pi_{\alpha} \\
& +{ }_{t} D_{b}^{2 \beta} q d \pi_{\beta}-{ }_{a} D_{t}^{\alpha} \pi_{\alpha} d{ }_{a} D_{t}^{\alpha} q+{ }_{t} D_{b}^{\beta} \pi_{\beta} d{ }_{t} D_{b}^{\beta} q \\
& +{ }_{t} D_{b}^{\alpha}\left(p_{\alpha}+{ }_{a} D_{t}^{\alpha} \pi_{\alpha}\right)+{ }_{a} D_{t}^{\beta}\left(p_{\beta}-{ }_{t} D_{b}^{\beta} \pi_{\beta}\right) \\
& \left.+{ }_{t} D_{b}^{2 \alpha} \pi_{\alpha}+{ }_{a} D_{t}^{2 \beta} \pi_{\beta}\right] d q-\frac{\partial L}{\partial t} d t
\end{aligned}
$$

This means that, the Hamiltonian is a function of the form

$$
H=H\left(q,{ }_{a} D_{t}^{\alpha} q,{ }_{t} D_{b}^{\beta} q, p_{\alpha}, p_{\beta}, \pi_{\alpha}, \pi_{\beta}, t\right)
$$

Thus, the total differential of this function reads as

$$
\begin{aligned}
& d H=\frac{\partial H}{\partial q} d q+\frac{\partial H}{\partial_{a} D_{t}^{\alpha} q} d_{a} D_{t}^{\alpha} q+\frac{\partial H}{\partial_{t} D_{b}^{\beta} q} d_{t} D_{b}^{\beta} q \\
& +\frac{\partial H}{\partial p_{\alpha}} d p_{\alpha}+\frac{\partial H}{\partial p_{\beta}} d p_{\beta}+\frac{\partial H}{\partial \pi_{\alpha}} d \pi_{\alpha}+\frac{\partial H}{\partial \pi_{\beta}} d \pi_{\beta} \\
& +\frac{\partial H}{\partial t} d t
\end{aligned}
$$

Comparing equation (17) with equation (19), we get the following Hamilton's equations of motion

$$
\begin{gathered}
\frac{\partial H}{\partial t}=-\frac{\partial L}{\partial t} \\
\frac{\partial H}{\partial p_{\alpha}}={ }_{a} D_{t}^{\alpha} q
\end{gathered}
$$

$$
\begin{gathered}
\frac{\partial H}{\partial p_{\beta}}={ }_{t} D_{b}^{\beta} q \\
\frac{\partial H}{\partial_{a} D_{t}^{\alpha} q}=-{ }_{a} D_{t}^{\alpha} \pi_{\alpha} \\
\frac{\partial H}{\partial_{t} D_{b}^{\beta} q}={ }_{t} D_{b}^{\beta} \pi_{\beta} \\
\frac{\partial H}{\partial \pi_{\alpha}}={ }_{a} D_{t}^{2 \alpha} q \\
\frac{\partial H}{\partial \pi_{\beta}}={ }_{t} D_{b}^{2 \beta} q
\end{gathered}
$$$$
\frac{\partial H}{\partial q}={ }_{t} D_{b}^{\alpha}\left(p_{\alpha}+{ }_{a} D_{t}^{\alpha} \pi_{\alpha}\right)+{ }_{a} D_{t}^{\beta}\left(p_{\beta}-{ }_{t} D_{b}^{\beta} \pi_{\beta}\right)
$$$$
+{ }_{t} D_{b}^{2 \alpha} \pi_{\alpha}+{ }_{a} D_{t}^{2 \beta} \pi_{\beta}
$$

\section{Illustrative Example}

We start with the following second order regular Lagrangian $[21,22]$.

$$
L=\frac{1}{2}\left[\ddot{q}^{2}-\dot{q}^{2}\right]
$$

In the presence of damping process $e^{\gamma t}$ the Lagrangian becomes [23].

$$
L=\frac{1}{2}\left[\ddot{q}^{2}-\dot{q}^{2}\right] e^{\gamma t}
$$

The corresponding fractional Lagrangian

$$
L=\left[\frac{1}{2}\left({ }_{a} D_{t}^{2 \alpha} q\right)^{2}-\frac{1}{2}\left({ }_{a} D_{t}^{\alpha} q\right)^{2}\right] e^{\gamma t}
$$

Making use of the Euler Lagrange equation (9), we obtain

$$
-{ }_{t} D_{b}^{\alpha}\left[\left({ }_{a} D_{t}^{\alpha} q\right) e^{\gamma t}\right]+{ }_{t} D_{b}^{2 \alpha}\left[\left({ }_{a} D_{t}^{2 \alpha} q\right) e^{\gamma t}\right]=0
$$

if $\gamma$ goes to zero and $\alpha=1$

we obtain the classical solution

$$
q(t)=C+D t-A \cos t-B \sin t
$$

The fractional canonical momenta read as

$$
\begin{gathered}
p_{\alpha}=-\left({ }_{a} D_{t}^{\alpha} q\right) e^{\gamma t}-{ }_{a} D_{t}^{\alpha}\left[\left({ }_{a} D_{t}^{2 \alpha} q\right) e^{\gamma t}\right] \\
p_{\beta}=0 \\
\pi_{\beta}=0
\end{gathered}
$$




$$
\pi_{\alpha}=\left({ }_{a} D_{t}^{2 \alpha} q\right) e^{\gamma t}
$$

Using equation (15), the Hamiltonian function is calculated as

$$
H=p_{\alpha a} D_{t}^{\alpha} q+\frac{1}{2}\left(\pi_{\alpha}\right)^{2} e^{-\gamma t}+\frac{1}{2}\left({ }_{a} D_{t}^{\alpha} q\right)^{2} e^{\gamma t}
$$

Thus, using equations (21-27), the equations of motion are

$$
\begin{gathered}
\frac{\partial H}{\partial p_{\alpha}}={ }_{a} D_{t}^{\alpha} q \\
\frac{\partial H}{\partial p_{\beta}}=0 \\
\frac{\partial H}{\partial{ }_{a} D_{t}^{\alpha} q}=p_{\alpha}+e{ }_{a}^{\gamma t} D_{t}^{\alpha} q \\
\frac{\partial H}{\partial{ }_{t} D_{b}^{\beta} q}=0 \\
\frac{\partial H}{\partial \pi_{\alpha}}=\pi_{\alpha} e^{-\gamma t}={ }_{a} D_{t}^{2 \alpha} q \\
\frac{\partial H}{\partial \pi_{\beta}}=0 \\
\frac{\partial H}{\partial q}={ }_{t} D_{b}^{\alpha}\left(p_{\alpha}+{ }_{a} D_{t}^{\alpha} \pi_{\alpha}\right)+{ }_{a} D_{t}^{\beta}\left(p_{\beta}-{ }_{t} D_{b}^{\beta} \pi_{\beta}\right) \\
+{ }_{t} D_{b}^{2 \alpha} \pi_{\alpha}+{ }_{a} D_{t}^{2 \beta} \pi_{\beta} \\
\left.\left.{ }_{a} D_{t}^{\alpha} q\right) e^{\gamma t}\right]+{ }_{t} D_{b}^{2 \alpha}\left[\left({ }_{a} D_{t}^{2 \alpha} q\right) e^{\gamma t}\right]
\end{gathered}
$$

This result is in exact agreement with that obtained by using fractional Lagrangian formulation, equation (31). Noting that one may obtain the classical solution, equation (32), if $\gamma$ goes to zero and $\alpha=1$ in equation (45).

\section{Conclusion}

As a continuation to previous works, we have used the Hamiltonian mechanics to obtain the fractional Hamiltonian formulation for nonconservative systems with second order Lagrangian. The fractional Euler Lagrange equations for these systems were derived. Also we obtained the fractional Hamiltonian for these systems and then, Hamiltonian equations of motion of these systems have been obtained in the same manner as those obtained by using the formulation of Euler Lagrange equations from variational problems; and it is observed that the Hamiltonian formulation is in exact agreement with the Lagrangian formulation.

\section{References}

[1] R. Metzler, J. Klafter, Physics Reports, 339, 1-77 (2000).

[2] S. G. Samko, A. A. Kilbas, and O. I. Marichev, Fractional Integrals and Derivatives: Theory and Applications, Gordon and Breach Science Publishers, Linghorne, PA, Switzerland; Philadelphia, PA, USA (1993).

[3] G. Zaslavsky, Hamiltonian Chaos and Fractional Dynamics. Oxford University Press, Oxford, (2005).

[4] B. J. West, M. Bologna, and P. Grigolini. Physics of Fractal Operators. Springer-Verlag, New York, (2003).

[5] F. Riewe, Physical Review E 53, 1890 (1996).

[6] F. Riewe, Phys. Rev. E 55, 3581 (1997).

[7] E. M. Rabei, T. S. Al halholy, and A, A. Taani, Turkish Journal of Physics. 28, (1) (2004).

[8] E. M. Rabei, T. S. Al halholy, and A. Rousan. International Journal of Modern Physics A, 19, 3083 (2004).

[9] E. M. Rabei, K. I. Nawafleh, R. S. Hijjawi, S. I. Muslih, and D. Baleanu, Journal of Mathematical Analysis and Applications 327, 891 (2007).

[10] O. P. Agrawal, An Analytical Scheme for Stochastic Dynamics Systems Containing Fractional Derivatives. ASME Design Engineering Technical Conferences. (1999).

[11] O. P. Agrawal, Journal of Applied Mechanics 68, 339 (2001).

[12] O. P. Agrawal, Journal of Mathematical Analysis and Applications 272, 368 (2002).

[13] D. Baleanu, S. Muslih, Physica Scripta 27, 105 (2005).

[14] D. W. Dreisi-gmeyer, P. M. Yoang, Journal of Physica A 36, 3297 (2003).

[15] M. Klimek, Journal of Physics 52, 1247, (2002).

[16] D. Baleanu, K. Tas, and S. Muslih, Journal of Mathematical Physics 47, 103503 (2006).

[17] E. H. Hasan, J. Asad, Journal of Advanced Physics 6 (3), 430 433 (2017).

[18] I. Podlubny, Fractional Differential Equations, Academic Press, New York, (1999).

[19] O. Jarab'ah, K. Nawafleh, and H. Ghassib, European Scientific Journal 9 (36), 132-154 (2013).

[20] H. Goldstein. Classical Mechanics (2nd edition). AddisonWesley, Reading- Massachusetts (1980).

[21] E. M. Rabei, E. H. Hasan and H. B. Ghassib, International Journal of Theoretical Physics 43, 1073 (2004).

[22] E. H. Hasan, Quantization of Higher-Order Constrained Lagrangian Systems Using the WKB Approximation, Ph.D. Thesis, University of Jordan (2004).

[23] O. Jarab'ah, E. H. Hasan, K. I. Nawafleh. European Scientific Journal 10 (9), 135-142 (2014). 